\title{
Sprachenwechsel und Koexistenz
}

\author{
Zur Rolle des Deutschen und Lateinischen in medizinischen \\ Fallsammlungen des 17. und 18. Jahrhunderts
}

\section{Einleitung}

Der in seiner Bedeutung für die Sprachgeschichte kaum zu überschätzende Übergang vom Gelehrtenlatein zu den europäischen Volkssprachen vollzog sich ausgesprochen disparat und keineswegs kontinuierlich. Der Prozess schritt in den verschiedenen Wissenschaftsbereichen unterschiedlich schnell voran. ${ }^{1}$ Als Gründe für die Verzögerung nennt Klein (2011a: 36-38) neben dem „konstitutiv konservativen Charakter vormoderner Gesellschaften“ die sozialdistinktive Funktion des Lateinischen sowie die in der zeitgenössischen Sprachdiskussion weit verbreitete Ansicht, dass das Deutsche für akademisch-gelehrte Abhandlungen überhaupt nicht geeignet sei. Dass es überhaupt zum Sprachenwechsel kam, führt Klein zum einen auf das Wirken Luthers und den „protestantischen Aufbruch“ (2011a: 39) zurück, zum anderen auf den frühen und häufigen Gebrauch des Deutschen in den anwendungsbezogenen Fächern von Schule und Universität.

Auf eine einfache Formel gebracht lässt sich davon sprechen, dass die Verdeutschung einzelner Wissenschaften desto schneller fortschritt, je mehr praktische Wirkungsmöglichkeiten in einer Disziplin vorhanden waren. (Klein 2011a: 40)

Wie aber darf man sich die Veränderung der Sprachenverhältnisse in einem spezifischen Kommunikationsbereich konkret vorstellen? Welche Übergangsformen bilden sich aus, und wie verändert sich der Umgang mit fremdsprachigen Elementen in den Texten? Der vorliegende Beitrag ${ }^{2}$ nimmt eine Textsorten-

1 Vgl. zur Entstehung und Entwicklung der deutschen Wissenschaftssprache z. B.: Polenz (2013: Kap. 5.11); Pörksen (1983, 2004); Kretzenbacher \& Weinrich (1995); Schiewe (1996); Klein (2011a, 2011b); zur Stellung des Deutschen in der Gegenwart vgl. Ammon (2015).

2 Für ihre wertvollen Hinweise und Anregungen danke ich den Tagungsteilnehmern, den Herausgebern und den beiden anonymen Gutachtern.

Bettina Lindner, Katholische Universität Eichstätt-Ingolstadt, Universitätsallee 1, 85072 Eichstätt, E-Mail: Bettina.Lindner@ku.de

Ә Open Access. (c) 2018 Bettina Lindner, publiziert von De Gruyter. (c) BY-NC-ND Dieses Werk ist lizenziert unter der Creative Commons Attribution-NonCommercial-NoDerivatives 4.0 Lizenz. https://doi.org/10.1515/9783110476958-011 
klasse ${ }^{3}$ in den Blick, die für eine Untersuchung dieser Entwicklungen besonders geeignet scheint, und zwar medizinische Fallsammlungen und die in ihnen enthaltenen ärztlichen Gutachten. Diese Texte können nicht nur als für ihre Zeit sehr typische Vertreter heilkundlicher Fachprosa ${ }^{4}$ gelten, sie liegen auch im Überschneidungsbereich zwischen medizinischer Praxis und akademischer Medizin und versprechen damit interessante Einblicke in die mit dem Sprachenwechsel ${ }^{5}$ verbundenen Vorgänge und Entwicklungen. ${ }^{6}$

Zwei Aspekte sollen im Folgenden im Mittelpunkt stehen: zum einen die Frage, inwiefern die sich ändernden sprachlichen Verhältnisse ihren Niederschlag in den Fallsammlungen finden; zum anderen soll eruiert werden, welche Formen und Funktionen fremdsprachiger Elemente nach dem vollzogenen Sprachenwechsel zu beobachten sind. Einer knappen Vorstellung des Formates und der darin vertretenen Textsorten folgen exemplarische Analysen einzelner Sammlungen und der in ihnen enthaltenen Texte.

\section{Die Fallsammlungen}

Medizinische Fallsammlungen gibt es bereits im 16. Jahrhundert, und zwar in ganz Europa. Unter dem Titel curationes, observationes oder historia veröffentlichten Mediziner, v. a. Stadt- und Gerichtsärzte, Sammlungen aus ihrer Praxis. Diese Sammlungen erschienen auf Latein und wurden zum Teil in die Volkssprache übersetzt. ${ }^{7}$ Im 17. und 18. Jahrhundert avancierten solche primär als Lehrwerke für das Medizinstudium konzipierten Fallsammlungen zu einem der

3 Den Ausführungen liegen die Begriffsdefinitionen von Wolfgang Heinemann (2000: 513514) zu Grunde.

4 Einen ersten Überblick zu den Spezifika dieser Fallsammlungen bietet der Medizinhistoriker Michael Stolberg (2007). Vor allem die Geschichts- und Literaturwissenschaft haben in den letzten Jahren medizinischen Fallsammlungen als wichtige Quellen für unterschiedliche Fragestellungen entdeckt, vgl. z.B. Fischer-Homberger (1988); Geyer-Kordesch (1990); Lorenz (1999); Müller \& Fangerau (2010); Pomata (2010, 2011); Daston (2011a, 2011b); Daston \& Lunbeck (2011); Berndt \& Fulda (2012); Behrens \& Zelle (2012); Wübben \& Zelle (2013); Düwell \& Pethes (2014).

5 Der Terminus ,Sprachenwechsel ${ }^{*}$ wird in der Forschung nicht einheitlich verwendet, sehr oft ist auch von ,language shift' die Rede.

6 Ähnliches ist sehr wahrscheinlich auch in anderen medizinischen Textsortenklassen des Spätmittelalters und der Frühen Neuzeit feststellbar. Weitere Untersuchungen werden zeigen, wie repräsentativ die hier beschriebenen Entwicklungen sind.

7 Zum Beispiel die Fallsammlung des Berner Stadtwundarztes Wilhelm Fabrys (1560-1634), die 1652 von Friedrich Greiffen übersetzt wurde. 
beliebtesten Formate medizinischer Fachliteratur. Bei diesen jüngeren Sammlungen sind es dann aber v. a. Universitätsmediziner, die als Herausgeber fungieren.

Es lassen sich zwei Typen von Sammlungen unterscheiden: solche, die in narrativer Form Krankengeschichten rekapitulieren, was vor allem für die älteren Sammlungen zutrifft; und es gibt solche, die den Charakter der originalen Dokumente - Anschreiben, Gerichtsgutachten, Consilia, Visa reperta - wahren und in kommentierter Form wiedergeben, was wiederum vor allem für die jüngeren Sammlungen ab dem letzten Drittel des 17. Jahrhundert gilt. Auf diesen zweiten Typus wollen wir uns hier konzentrieren.

Die meisten Fälle stammen von akademisch gebildeten Ärzten aus dem protestantisch geprägten ostmitteldeutschen Raum, wo sich die Gerichtsmedizin am frühesten und differenziertesten entwickelte. Als intendierte Adressaten werden oft junge Ärzte und Medizinstudenten genannt. Die Auswertung von Pränumeranden- und Subskribentenverzeichnissen bestätigt, dass es hauptsächlich Ärzte, Apotheker, Chirurgen, Juristen und Pfarrer waren, die sich für die Sammlungen interessierten (vgl. Lorenz 1999: 37).

Besonderen Wert - nicht nur für sprachhistorische Fragestellungen - haben die uns durch die Fallsammlungen erhaltenen, im Wortlaut abgedruckten, medizinischen Fachtextsorten, nämlich:

1. Consilia: Texte instruktiver Natur, die darauf ausgelegt sind, das Verhalten ihrer Leser, Patienten und anderer Heilkundiger, zu beeinflussen. Sie legen Strategien zur Ansteckungsvermeidung nahe oder geben Empfehlungen für den Fall, dass eine Ansteckung bereits erfolgt ist.

2. Gerichtsmedizinische Gutachten: Stellungnehmende Texte mit dominierend argumentativer Vertextungsstrategie, die als Entscheidungsgrundlage für den Richter gedacht sind.

3. Relationes oder Visa reperta: Texte deskriptiver Prägung, welche die im Auftrag der Obrigkeit vorgenommene Untersuchung eines Kranken oder Leichnams dokumentieren, Letzteres häufig auch unter Einschluss der Obduktion.

Die Zuordnung zu einer der eben beschriebenen Textsorten ist allerdings nicht immer eindeutig - es ergeben sich zahlreiche Überschneidungen und Mischformen. ${ }^{8}$

8 Vgl. meine Erlanger Dissertation Medizinische Gutachten des 17. und 18. Jahrhunderts. Sprachhistorische Untersuchungen zu einer Textsortenklasse (Lindner 2018). 


\section{Zur Sprachwahl in den Sammlungen}

Die meines Wissens erste Sammlung, die deutschsprachige Originalgutachten abdruckt, ist die Medicina Critica von Paul Ammann, die 1670 erschien. Titel, Vorwort und Kommentierung der Gutachten sind lateinisch, während Anfragen und Antworten der medizinischen Fakultät größtenteils auf Deutsch verfasst werden. Wie ist dieses Nebeneinander von volkssprachlichen Gutachten und lateinischer Kommentierung zu erklären?

Dass man, im Gegensatz zu den älteren Sammlungen, originale Dokumente abdruckt, ist der generellen Aufwertung der Empirie in der akademischen Medizin des 17. und 18. Jahrhunderts geschuldet (vgl. hierzu Stolberg 2003). Zwar beruft man sich immer noch gerne auf die antiken Autoritäten, wie Hippokrates und Galen, immer wichtiger wird aber zusätzlich die selbst gewonnene Erfahrung. Und die lässt sich am besten einbringen, wenn authentische Fälle geschildert werden. Das wörtliche Abdrucken von Originaldokumenten dient freilich auch dazu, die Glaubwürdigkeit des Herausgebers zu erhöhen, analog zu den zunehmend präziser werdenden Quellenangaben bei Zitaten (vgl. Lindner 2015), die die Nachprüfbarkeit erhöhen.

Dass es deutschsprachige Texte sind, die abgedruckt werden, liegt daran, dass die medizinische Praxis schon wesentlich früher volkssprachlich geprägt war als die akademische Medizin. Zu erklären ist das nach meiner Einschätzung auch mit der Situation auf dem medizinischen Markt: Akademische Ärzte konkurrierten und kooperierten mit einer Reihe eher handwerklich geprägter Heilkundiger - Chirurgen, Bader, Apotheker und Hebammen -, die mit den Patienten in der Volkssprache kommunizierten. Wollte man hier bestehen und nicht zuletzt ökonomisch profitieren, blieb gar nichts anderes übrig, als ebenfalls auf Deutsch zu kommunizieren. Das zeigt sich auch an den einzelnen in den Sammlungen vertretenen Textsorten. Die Untersuchung und Obduktion eines Leichnams wurde im Allgemeinen von einem (lateinunkundigen) Chirurgen und einem studierten Physikus durchgeführt und anschließend in einem Visum repertum / Sektionsbericht festgehalten. Da beide Experten gleichermaßen für das Dokument verantwortlich zeichneten, lag die Wahl der Volkssprache sehr nahe.

Relativ früh volkssprachlich geprägt war auch die Kommunikation in der Verwaltung, in deren Zusammenhang die Gerichtsgutachten entstanden. Die Verfasser dieser Stellungnahmen, einzelne Mediziner oder medizinische Kollegien und Fakultäten, entschieden sich daher im Dienste der Verständlichkeit ebenfalls für das Deutsche. Gleiches gilt für die dritte Textsorte: Da weder die betuchte städtische Oberschicht noch breitere Bevölkerungsschichten auf La- 
tein erreicht werden konnten, waren auch die meisten Consilia in der Volkssprache verfasst.

Gleichzeitig hielt man aber im akademischen Umfeld am Statussymbol Latein fest. Wer lateinisch sprach und las, gehörte, wie Klein (2011a: 37) zu Recht betont, einem besonderen sozialen Raum mit deutlich elitärem Charakter an und ,bekräftigte diese Zugehörigkeit bei jeder Lektüre oder Produktion lateinischer Texte“. Sicherlich liegt hier einer der Gründe, warum auch und gerade die akademische Medizin so lange am Lateinischen festhielt: Man war sich des Status als vollwertiges universitäres Fach sehr bewusst und sehr darauf bedacht, diesen Status zu betonen, wozu die lateinische Sprache besonders gut geeignet erschien. Und so verwundert es auch nicht, dass in Ammanns Lehrbuch die Kommentierung der Fälle, die der Einordnung in die medizinische Systematik dient, auf Latein erfolgte. Man trennte sprachlich streng zwischen der Praxis bzw. Empirie auf der einen Seite und akademischer Reflexion auf der anderen.

Dass sich dieser ausgeprägte Gegensatz nicht aufrecht erhalten ließ, ist hinlänglich bekannt. Als nächster Schritt kann die Herausgabe einer Sammlung in zwei Versionen gelten, einer deutschsprachigen und einer lateinischen. So erschienen 1706 zwei in Inhalt und Struktur identische Ausgaben der Medicina Forensis von Friedrich Zittmann, die sich aber in Titelblatt und Vorwort unterscheiden. Beide stellen zwar lateinische Gutachten neben deutschsprachige; Titelblatt und Vorwort sind aber einmal auf Deutsch und einmal auf Latein verfasst. Dass studierte Ärzte nicht immer die lateinische Variante vorzogen, zeigt der Bibliotheksbestand des Nürnberger Leibarztes Christoph Jacob Trew, ${ }^{9}$ der die Sammlung Zittmanns in der deutschen Version enthält. ${ }^{10}$ Der (lateinisch-)deutsche Titel ist im Übrigen keine Übersetzung des rein lateinischen, er unterscheidet sich formal wie inhaltlich. Während der lateinische Titel durchgehend in Antiqua gesetzt ist und insgesamt recht schlicht anmutet, wechseln sich Fraktur und Antiqua bei der deutschen Ausgabe ab. Letztere ist in Schwarz-Rot gehalten; neben ganz in Versalien gesetzten Teilen wie dem Haupttitel sind einzelne Wörter auch mit Schmuckinitialen versehen. ${ }^{11}$ Der volkssprachliche Titel ist dank zusätzlicher Angaben zu Inhalt und Struktur

9 Zur Person und Sammlung Christoph Jacob Trews vgl. Schnalke (1995), zu seiner umfangreichen Korrespondenz Schnalke (1997) und Ruisinger (2008).

10 Vgl. die Sammlung Trew in der UB Erlangen-Nürnberg, Signatur H61/4 TREW.Q 462.

11 Dass sich unterschiedliche Schriftgrößen, Schrifttypen und Farben abwechseln, ist durchaus üblich, wie die Auswertung des Corpus von Titelblättern in der Untersuchung von Götz (2011: 74) zeigt. 
deutlich länger als der lateinische (vgl. die folgende Tabelle). Während Letzterer nur die enthaltenen Texte, deren Herkunft und Entstehungsjahr, den Verfasser, dessen Ämter und Funktionen, Ankündigung eines Vorwortes und Registers, Druckort und Jahr sowie die Angabe, dass das Werk von der Societät bezahlt worden sei, auflistet, wurde der lateinisch-deutsche Titel ergänzt. Offenbar hielt man es für notwendig, zusätzlich werbewirksam wertende Bemerkungen zur Qualität der Antwortschreiben zu machen, die „hoch vernünftig“ seien und sich auf „fchwere/ 3weiffelhaffte und feltene“ Fällen bezögen. Verkaufsfördernd wirken sollten vermutlich auch die Betonung der Neuheit dieser Art von Büchern sowie der Hinweis auf die enthaltenen Kupfer und das Register. Im lateinisch-deutschen Titel wird außerdem der intendierte Leserkreis genannt, der im Vergleich $\mathrm{zu}$ anderen Sammlungen relativ weit gefasst ist und nicht nur Mediziner und Wundärzte, sondern auch Rechtsgelehrte und Theologen einschließt. Dass eine deutsche Ausgabe auch zu diesem Zeitpunkt noch nicht vorbehaltlos unterstützt wurde, legen die Angaben zur Finanzierung nahe: Die lateinische Version wurde offenbar von der gesamten Societät finanziert, während die Kosten für die deutsche Ausgabe nur von einigen Mitgliedern getragen wurden.

\begin{tabular}{|c|c|}
\hline Lateinischer Titel & Lateinisch-deutscher Titel \\
\hline 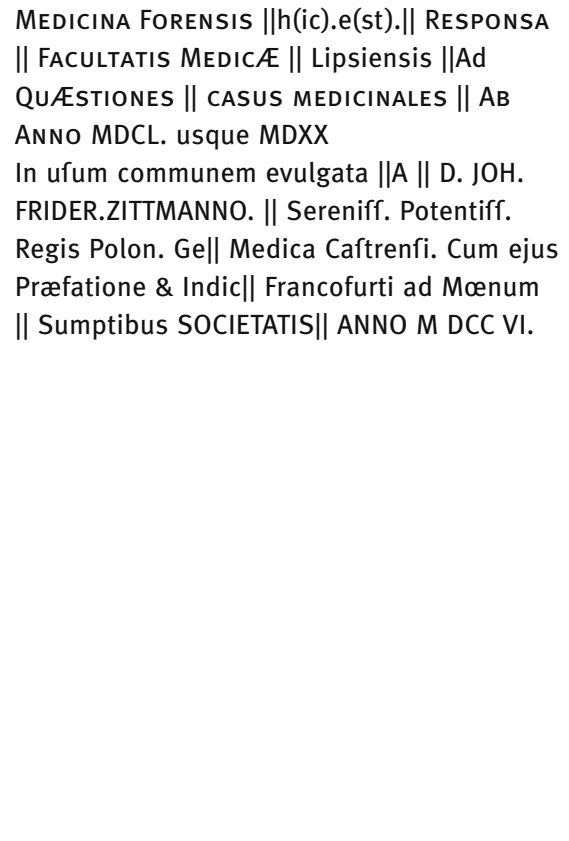 & 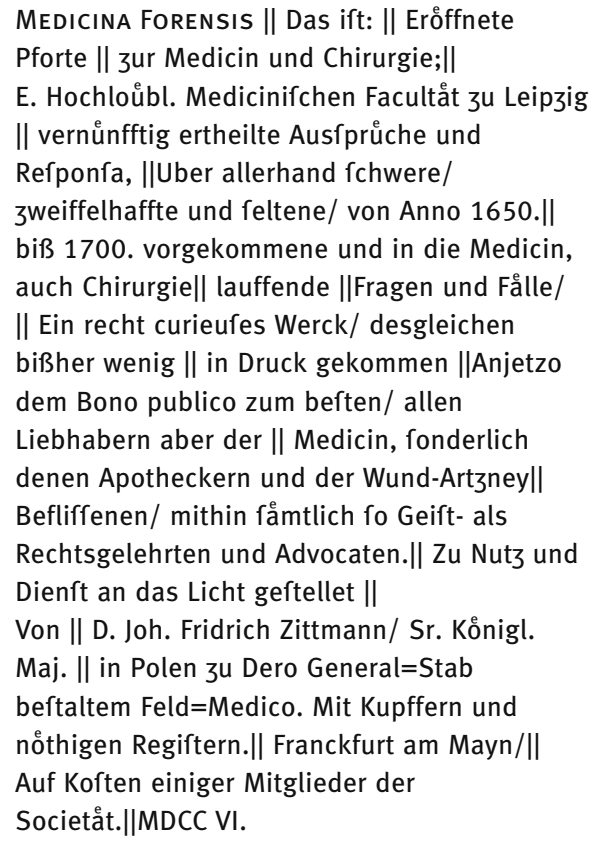 \\
\hline
\end{tabular}


Lateinische Ausgaben hatten gegenüber den volkssprachlichen den unschätzbaren Vorteil, dass auch ein internationales Publikum erreicht werden konnte. Dass ihre Werke auch im Ausland gelesen werden konnten, war insbesondere den berühmteren Medizinern wie Georg Ernst Stahl oder Friedrich Hoffmann wichtig. Der deutlich geringere Wirkungskreis volkssprachlicher Texte dürfte hemmend auf den Sprachenwechsel gewirkt haben.

Um die Mitte des 18. Jahrhunderts haben sich die Sammlungsherausgeber endgültig von der lateinischen Kommentierung verabschiedet. Gleichzeitig setzen sich volkssprachliche Titel durch. Die Fallsammlungen entsprechen damit ganz dem allgemeinen Trend - denn generell gilt, dass auf dem Buchmarkt um 1750 lateinische Publikationen stark rückläufig waren, während gleichzeitig volkssprachliche Werke zunahmen (vgl. Pörksen 1983).

\section{Zum Verhältnis von Deutsch und Latein in den Gutachten}

Um 1750 ist der Sprachenwechsel in den Fallsammlungen also für wichtige Textteile vollzogen - Titel, Vorworte, Kommentare etc. sind auf Deutsch verfasst. Schon ein flüchtiger Blick in die Texte zeigt aber, dass das Lateinische keineswegs gänzlich verschwunden ist. Lateinische Elemente sind vielmehr immer noch ein absolut typisches Merkmal medizinischer Fachprosa. Welche Formen und Funktionen kommen diesen nun fremdsprachigen Elementen in den volkssprachlichen Texten zu? Werfen wir zunächst einen Blick in die zeitgenössischen Textsortenanleitungen, die Ratschläge erteilen, wie medizinische Gutachten zu verfassen sind. Welche Meinung vertreten sie hinsichtlich der Sprachwahl und fremdsprachiger Elemente?

Seit dem Anfang des 18. Jahrhunderts wurden solche Anleitungen verstärkt nachgefragt und es erschienen diverse Schriften monographischen Charakters. Aber auch die Herausgeber der Sammlungen reagierten in ihren Vorworten und Kommentaren auf das Bedürfnis der Leser nach Hilfestellung und Anleitung. Verglichen mit anderen Textsorten haben sich - ein Glücksfall für die Forschung - verhältnismäßig viele anleitende Texte und metakommunikative Äußerungen erhalten.

Die Wahl der Sprache spielt in den Anleitungen keine Rolle mehr - man muss die Einstellung zu dieser Frage also indirekt aus den Stilvorgaben erschließen. Die Vorgaben für medizinische Gutachten unterscheiden sich dabei keineswegs von den rhetorischen Leitprinzipien eines Gottsched oder Adelung. Wie diese treten auch die Verfasser der Anleitungen für Prinzipien wie ,Deut- 
lichkeit', ,Klarheit‘ und ,Kürze‘ ein. ${ }^{12}$,Deutlichkeit‘ bezieht sich auch auf den lexikalischen Bereich, und unter dieser Prämisse wird der Gebrauch von Fremdwörtern insgesamt kritisch gesehen.

Die älteste deutschsprachige Textsortenanleitung, eine 1713 herausgegebene Übersetzung einer französischen Anleitung von Jean Devaux, empfiehlt, auf „undeutliche[ ]/barbarifche[ ] und fcholaftifche[ ] grillen“ (Devaux 1713: 20) zu verzichten. Auch die 1767 erschienene Anleitung von Gottlieb Büttner (1767: 2-3) ermahnt angehende Ärzte, in „Obductions-Atteftata“ nicht „lauter lateinifche[ ], ja gar griechifche[] Ausdrücke[ ]“ zu gebrauchen, sondern diese „follen und můßen [...] nothwendig deutfch gegeben werden [...]“.

Von einer streng puristischen Haltung, die jedes Fremdwort verbietet, ist man in den Anleitungen aber weit entfernt. Denn auch die Verfasser solcher Instruktionen müssen sich selbst den Vorwurf des übermäßigen Fremdwortgebrauchs gefallen lassen. In einer Rezension der Allgemeinen Deutschen Bibliothek $(A D B)^{13}$ kritisiert der Rezensent den eben zitierten Gottlieb Büttner wie folgt: „Er ift wohl eben nicht fehr Meifter im guten Lehrftile, bedient fich auch im Unterrichte einer Menge lateinifcher Worte ohne Noth." ${ }^{14}$

Die Textsortenanleitungen vertreten also eine gemäßigt fremdwortkritische Haltung. Wie sieht es aber in den Texten aus? Die Sammlungsherausgeber tun sich mit der in den Anleitungen formulierten Forderung, auf Fremdwörter gänzlich zu verzichten, offenbar schwer. Sie sichern sich zum Teil schon in den Vorworten gegen den Vorwurf des übermäßigen Fremdwortgebrauchs ab und/oder verweisen auf ergänzende Glossare, die das Verständnis erleichtern sollen:

Weilen aber auch die termini medico-technici, wenigen die die Medicin nicht ftudiret, bekannt, und doch um das Werk nicht obfcur zu machen, nicht fchicklich in das teutfche zu ůberfetzen feyn, fo habe eine Erlåuterung gedachter terminorum, und noch ein vollkommen Regifter mit anhăngen follen. (Hasenest 1755: Vorrede unpaginiert)

Man kann diefes Collegium zwar nicht vor vo̊llig ins Deutfche ůberfet3t ausgeben, allermaffen man durchgehends die Terminos technicos in ihrer erften Kleidung laffen můffen:

12 Vgl. allgemein zu den Stilprinzipien in den Wissenschaften auch Kretzenbacher (1995).

13 Die von Friedrich Nicolai seit 1765 herausgegebene Rezensionszeitschrift Allgemeine Deutsche Bibliothek erschien vierteljährig. Jedes Jahr wurden zwei Bände veröffentlicht, jeder Band in zwei Stücken. Dank des an der Universität Bielefeld angesiedelten Projekts „Retrospektive Digitalisierung wissenschaftlicher Rezensionsorgane und Literaturzeitschriften des 18. und 19. Jahrhunderts aus dem deutschen Sprachraum“ kann die ADB online durchsucht werden, vgl. http://www.ub.uni-bielefeld.de/diglib/aufklaerung/.

14 Nach diesem eher negativen Urteil schlägt der Rezensent versöhnlichere Töne an, denn er räumt ein, dass in dem Werk auch viel Gutes und nicht nur Gemeines zu finden sei (ADB 1770, Bd. 13, St. 2, 508). 
da es aber nicht die Absicht hat, daß es viele Leute, welche der Lateinifchen Sprache nicht måchtig find lefen follen, fondern nur vor diejenigen, die die Lateinifche, ja! Medicinifchlateinifche Sprache verftehen, gefchrieben ift und diefe hinlånglich erkennen werden, daß die emphatifche Expreffiones ohnmo̊glich ůbergehen ko̊nnen, fo wird folches vor keinen Fehler gerechnet werden. (Stahl 1733: Vorrede unpaginiert)

Wie diese Bemerkungen schon vermuten lassen, gebrauchen die Ärzte in ihren Gutachten tatsächlich recht häufig Fremdwörter, wie die folgenden Beispiele veranschaulichen:

1. Von der Wunde des cranii linker Hand gieng eine fiffur oder Spal=tung eines Zolls lang bis an die [...] (Hasenest 1757: 184)

2. Die ůbrigen humores zu temperiren und in ein beffer Gefchick zu bringen [...] (Gohl 1735: 241)

3. So geben wir demfelben zu verlangter Antwort, daß gedachter Frau Patientin ietziger Affectus nichts anders, als Arthritis und Con-tractura a fero fangvinis vifcido \& acido-acri oriunda zu nennen: (Budaeus 1735: 4)

4. [...] hat es leicht gefchehen ko̊nnen, daß einige fubtiliffima vafa lymphatica dilacerirt und eine extravafirte lympha circa thalamos nervorum opticorum aufgetreten, welche, nachdem fie durch Weile der Zeit eine arfcidi-tăt $a c$ quirirt, durch Drückung des nervi optici zu Anfangs eine Dunckelheit, und nachmahlen gar die amaurofin oculi finiftri verurfachet. (Gohl 1735: 59-60)

Überwiegend handelt es sich um Fachtermini lateinischen oder griechischen Ursprungs, die Krankheiten, Symptome, anatomische Gegebenheiten, Anomalien, Pharmazeutica oder Therapien bezeichnen. Deutlich ist auch die Verankerung in dem auch im 18. Jahrhundert noch gültigen System der Humoralpathologie.

Meist sind es lexikalische Transferenzen, d.h. die Textproduzenten gebrauchen die fremdsprachigen Lexeme und Phraseologismen nur kursorisch in deutschen Sätzen. Die fremdsprachige Flexion wird teils beibehalten und der Syntax angepasst, teils durch indigene Morpheme ersetzt (z.B. arfcidi-tăt, acquirirt, dilacerirt [4]). Wie in dieser Zeit üblich sind die als fremdsprachig empfundenen Elemente typographisch in Antiqua ausgezeichnet (in obigen Belegen kursiv), die Type wechselt zum Teil mitten im Wort (vgl. hierzu Schulz 2012; Polenz 2013: 65). Die Beispiele sind in ihrer Kürze durchaus typisch. Vereinzelt und gegen Ende des Untersuchungszeitraumes immer seltener flechten die Verfasser längere fremdsprachige Abschnitte in ihre Texte ein. So untermauert etwa Troppanneger (1733: 68-69) seine Argumentation mit einem lateinischen Zitat Johann Bohns, eines frühen Forensikers: 
Bohnius de off. med. dupl. Part.I. Cap. 14. de Purgatione. hiervon faget, da diefer das letztere Tempus matutinum ad ordinarium modum propinandi emetica rechnet; allwo er faget: Ordinarie Jejunis vomitoria exhibentur, illis tamen qui difficulter \& cum fingulari moleftia hinc moventur, poft paftum paulo propinari valent, pro evacuatione facilitanda.

Fachtermini erfüllen eine ähnliche Funktion wie Zitate. Da die medizinische Terminologie erst gegen Ende des 19. Jahrhunderts definiert und vereinheitlicht wurde, ist der Gebrauch lateinischer und griechischer Fremdwörter nicht unbedingt im Dienste präziserer Benennungen zu sehen. Sie dienen wohl vor allem als Distinktionsmerkmal, das die Zugehörigkeit zu einer bestimmten medizinischen Schule demonstriert und überhaupt Fachkompetenz beweisen soll.

Der Gebrauch fremdsprachiger Fachtermini kann mithin als Glaubwürdigkeitssignal interpretiert werden. Die Verfasser scheinen dabei aber genau abzuwägen zwischen dem Erzeugen eines forciert wissenschaftlichen Erscheinungsbildes und der Verständlichkeit ihres Gutachtens. Die fremdsprachigen Einsprengsel sind wohl dosiert, und die Verfasser bedienen sich verschiedener Verfahren, um die Verständlichkeit ihrer Texte zu sichern. Dazu zählen Glossare wie das von Johann Georg Hasenest. Die Erläuterung der Fachtermini wird damit extern, außerhalb des Textes geleistet (vgl. Hasenest 1755). Es lassen sich aber auch innerhalb der Texte Bemühungen erkennen, die Verständlichkeit zu sichern. Betrachten wir dafür die folgenden Beispiele:

5. in dem Pericardio oder Hert3=Kammer fande fich gant3 nichts von der gewo̊hn=lichen Feuchtigkeit, [...] (Budaeus 1737: 77)

6. [...] vertebræ colli oder das Genicke ebenfals auswendig / und nach der Oeffnung inwendig gant3 natůrlich und ohnverletzet; [...] (Petermann 1709: 57)

7. Die Leber war von un=gewo̊hnlicher Gro̊ffe durchwachfen mit vielen unnatůrlichen Gewåchfen und Hårtigkeiten, atheromata oder melicerides genennt; (Gohl 1735: 36-37)

8. Von der Wunde des cranii linker Hand gieng eine fiffur oder Spal=tung eines Zolls lang bis an die [...] (Hasenest 1757: 184)

9. Als der Unterleib geoffnet wurde, fo floß eine Menge gelbes Blutwaffer aus; die großen Gefåße, nem=lich die Hohlader und die Hauptarterie Aor-ta, waren von Blut ganz leer. (Metzger 1781: 13)

10. [...] einen Stich mit einem Meffer am Hals auf der rechten Seite neben der grof=fen Flechfe des Nackens (tendo ceruicis) bekom=men, welcher fo tief drang, daß davon nicht allein die Halsader (vena iugularis) und Speiferôhre (Oefophagus) getroffen, [...] (Ruef 1777: 119)

Das wichtigste Erschließungsverfahren besteht dabei in der Angabe von volkssprachlichen Bezeichnungsalternativen. So können etwa der fremdsprachige Terminus und seine deutsche Entsprechung durch oder koordiniert werden 
(vgl. 5, 6, 8). Eine weitere Möglichkeit, dem Leser die Terminologie näher zu bringen, besteht darin, den Fachterminus erst in einem (nachgestellten) Attribut zu bieten, wie in (7) oder (9). Der Vorteil dieser Darstellung liegt darin, dass zusätzliche Informationen über die Begriffshierachie, also zu Über- bzw. Unterordnungsverhältnissen vermittelt werden können. Das letzte Beispiel (10) ist ein Beleg für das frühe Bemühen, auch volkssprachliche Fachtermini zu verwenden. Dass man dem Deutschen dann doch noch nicht ganz über den Weg traute, zeigt sich daran, dass man die lateinische Bezeichnung in Klammern dahinter gesetzt hat.

Neben diesen Erklärungen, lassen sich auch noch andere, subtilere Strategien der Verdolmetschung ausmachen, wie das folgende Beispiel zeigt.

Wenn wir nun auf die vorgelegte Frage: an partus hic fu-rit vitalis? unfer gegründetes Bedencken ertheilen follen; fo můffen wir zufoürderft zu defto gründlicherer und gnugfamer Information Meldung thun: daß das prædicatum vitalis, wenn es einem foetui beygeleget wird, auf dreyerley Art ko̊nne verftanden werden. Denn erftlich wenn fich das punctum faliens fub formatione zeiget, oder der motus cordis \& progreffus humorum die erften Monate fich an=hebet und die gantze Machine und deren partes folidæ dadurch nach und nach nutriret und expandiret werden; fo ift vita verhanden und der embryo vitalis, obgleich die Mutter die ihn trågt, felbft davon noch nichts fůhlet und weiß. Wenn weiter die partes externæ und ar-tus ratione offium, mufculum, nervorum \& tendinum, volllig ge=bildet find, welches meiftentheils um die Helffte gefchehen ift; fo kan fich alsdenn der formirte foetus felbft bewegen und es erånget fich eine em= pfindliche Bewegung im Mutterleibe, dadurch eine Frau vo̊llig verfi=chert wird von der Schwangerfchafft und daß ihre Leibes=Frucht lebe, oder vitalis fey. Wenn aber endlich das Kind nicht allein vôllig ge=ftaltet fondern auch nunmehro alle partes an denfelben zu folcher Fe=ftigkeit und Vollkommenheit gediehen, daß es nicht mehr von der Mut=ter Blut ernåhret werden darf, fondern extra uterum in der Lufft von der ihm gereichten Milch und Speife leben, dauren und nach und nach wachfen und vollkommner werden kan; fo wird ein folcher fotus gleich=falls, und 3war im eigentlichen Verftande, vitalis genennet. (Hoffmann 1726: 181; Fettdruck durch die Verf.)

Zunächst wählt der Verfasser, ein akademisches Kollektiv, mit foetus einen lateinischen Fachterminus, wechselt dann zu griechisch embryo, um schließlich zu ergänzen: „obgleich die Mutter, die ihn trågt noch nichts von ihm fühlet oder weiß“. Dank dieses Zusatzes kann auch ein medizinischer Laie erschließen, dass mit foetus und embryo der menschliche Nachwuchs im Frühstadium gemeint ist. Im Übrigen wird auch durch die textliche Wiederaufnahme mit „Leibes-Frucht“ und „Kind“ klargestellt, welche Bedeutung die beiden Fachtermini haben. Das heißt, der Leser kann, selbst wenn er einige Termini auf Anhieb nicht versteht, deren semantischen Gehalt entweder aus dem Kontext erschließen oder aus seinem Weltwissen ergänzen.

Welche Funktionen haben die fremdsprachigen Elemente in den Gutachten? Sie dienen der präzisen Bezeichnung von Krankheiten, anatomischen Ge- 
gebenheiten etc. Außerdem haben wir schon auf ihre sozialdistinguierende Funktion hingewiesen, und darauf, dass sie identitätsstiftend eine bedeutende Rolle spielen. Eng damit verbunden ist das Bedürfnis, Kompetenz unter Beweis zu stellen. Eine weitere wichtige Funktion wird am folgenden Beispiel deutlich. Es handelt sich um ein Gutachten, das für ein Konsistorialgericht verfasst wurde und eine Ehestreitigkeit betrifft. Eine Frau möchte sich von ihrem Mann wegen dessen Impotenz scheiden lassen. Das Gutachten ist auf Deutsch verfasst. Als aber das anstößige Faktum zur Sprache kommt, verwendet der Verfasser überwiegend lateinische Termini und Wendungen:

Weilen aber auch die erectio membri nicht genug, fondern die gantze Sache auf den coitum und emiffionem \& imiffionem femi-nis virilis in partem muliebrem ankömmet, fo kan auch folches wol falva honeftate \& verecundia hier gefchehen, wenn auf vorerwehnte Art die erectio membri bey Titio erhalten würde, derfelbe auch fo fort, folches der Marcellæ, jedoch in einem neben Gemach, ohne anderer $\mathrm{Ge}=$ genwart, alfo applicirte, daß entweder Marcella die immiffionem fe-minis ipfius empfinden oder folcher doch in vagina uteri ipfius befun=den werden könne: Allermaffen auch in fœcundo coitu femen maris tantum circa uteri orificium internum, effundiret wird, und der meifte Theil deffelben bald wiederum abgehet, [...] (Alberti 1725: 11)

Der Wechsel zur Gelehrtensprache dient also offenbar der Vermeidung von Peinlichkeiten und der Wahrung des Anstandes - ein Befund, der, das zeigen weitere Beispiele, sich durchaus generalisieren lässt.

\section{Schluss}

Wie lassen sich die Ergebnisse nun pointiert zusammenfassen? Es ist der Frage nachgegangen worden, wie sich der Wechsel von der Gelehrten- zur Volkssprache in einem begrenzten Kommunikationsbereich vollzogen hat. Medizinische Fallsammlungen erwiesen sich aufgrund ihrer Situierung im Überschneidungsbereich von Wissenschaft und Praxis als ein für diese Problemstellung besonders geeigneter Gegenstand. Deutschsprachige Gutachten lassen sich ab dem letzten Drittel des 17. Jahrhunderts nachweisen - als die Empirie in Medizin und anderen Wissenschaften eine generelle Aufwertung erfuhr. Sie stehen zu diesem Zeitpunkt aber noch neben lateinischen Texten. Der Wechsel vollzog sich langsam und diskontinuierlich, und es lassen sich diverse Übergangsformen beobachten. In der ersten Phase trennen die Herausgeber noch streng zwischen volkssprachlicher Empirie - den verschiedenen Gutachtentypen und der akademischen Reflexion auf Latein. Ein weiterer Schritt war die Herausgabe zweier in Inhalt und Struktur zwar identischer, sprachlich aber ab- 
weichender Versionen der gleichen Sammlung. Seit etwa 1750 wurden die kasuistischen Lehrwerke dann gänzlich auf Deutsch verfasst und folgten damit dem allgemeinen Trend in den Naturwissenschaften.

Auch nach dem vollzogenen Sprachenwechsel spielten lateinische und griechische Elemente noch eine bedeutende Rolle. Zwar vertraten die Textsortenanleitungen zur Verfertigung medizinischer Gutachten eine fremdwortkritische Haltung gemäß den rhetorischen Stilprinzipien der Zeit, die Ärzte taten sich aber sichtlich schwer, diesen Vorgaben vollständig zu entsprechen. In der Terminologie oder in Gestalt von Zitaten flossen immer wieder fremdsprachige Elemente ein, in der unverkennbaren Absicht, Glaubwürdigkeit und hohe fachliche Kompetenz zu vermitteln. Zugleich waren die Mediziner aber auch darauf bedacht, das Textverständnis nicht zu gefährden und Verfahren zu benutzen, die es auch dem lateinunkundigen Leser ermöglichten, der Argumentationslinie $\mathrm{zu}$ folgen.

\section{Quellen}

Alberti, Michael (1725): Systema jurisprudentiae medicae, quo casus forenses, a jctis et medicus decidendi, explicantur omniumque facultatum sententus confirmantur, in partem dogmaticam et practicam partitum, casibus, relationibus jurididis et medicis forensibus specialibus illustratum [...]. Pars posterior practica sive casuistica et forensis. Halle.

Anonym (Jean Devaux) (1713): Die Kunst Chirurgische Berichte und Wund=Zettul abzufassen. Aus dem Frantzösischen ins Teutsche übersetzt. Budißin: Johann George Hüneln.

Ammann, Paul (1670): Medicina Critica Sive Decisoria, Centuria Casuum medicinalium in concilio Facult. Med Lipf. Antehac refolutorum, comprehenfa, nunc vero in Phyficorum, Practicorum, Studioforum, Chirurgorum alioumq, ufum notabilem, collecta, correcta, \& variis Difcurfibus aucta, a D. Paulo Ammanno. Erfurt.

Budaeus, Gottlieb (1735): Miscellanea medico chirurgica, practica et forensia; Worinnen Allerhand Medicinische und Chirurgische Casvs, Relationes, Renvnciationes, Jvdicia, Consilia, Responsa, \&c. Enthalten: Fortgesetzt im Fünfften Theile. Leipzig, Görlitz: Johann Gottlob Laurentius.

Budaeus, Gottlieb (1737): Miscellanea medico chirurgica, practica et forensia; Worinnen Allerhand Medicinische und Chirurgische Casvs, Relationes, Renvnciationes, Jvdicia, Consilia, Responsa, \&c. Enthalten: Fortgesetzt im Sechsten Theil. Leipzig, Görlitz: Johann Gottlob Laurentius.

Büttner, Christoph Gottlieb (1767): Anweisung für angehende Arzeneybeflißene, worauf sie bey Ausstellung eines Obductions-Attestes über tödtliche Verletzungen mit Acht zu geben haben. Königsberg: Johann Jakob Kanter.

Gohl, Johann Daniel (1735): Medicina practica, clinica et forensis, sive collectio casvvm rariorvm ac notabiliorvm medico-clinicorvm, chirurgicorvm ac forensivm, partim responsis ac epicrisibvs, partim sectionibvs anatomicis et dispositionibvs illustratorvm [...]. Leipzig. 
Greiffen, Friderich (1652): Herrn Guilhelmi Fabricii von Hilden Längst begehrte vollkommende Leib= und Wundartzney. Aus dem Lateinischen in Hoch Teutsche Sprach ubersetzet: Durch Friderich Greiffen/ Med. Cand. Frankfurt a. M.: Johann Beyers.

Hasenest, Johann Georg (1755): Der Medicinische Richter oder Acta Physico-Medico Forensia Collegii Medici Onoldini; Von Anno 1735 biß auf dermalige Zeiten zusammen getragen, hier und dar mit Anmerckungen, Dann Mit einer deutlichen Erläuterung der medicinischen Kunstwörter und vollständigen Register versehen. Onolzbach.

Hasenest, Johann Georg (1757): Der Medicinische Richter oder Acta Physico-Medico Forensia Collegii Medici Onoldini. Dritter Theil. Von Anno 1735 biß auf dermalige Zeiten zusammen getragen, hier und dar mit Anmerckungen, Dann Mit einer deutlichen Erläuterung der medicinischen Kunstwörter und vollständigen Register versehen. Onolzbach.

Hoffmann, Friedrich (1726): Medicina Consultatoria, Worinnen Unterschiedliche über einige schwehre Casus ausgearbeitete Consilia und Responsa Facultatis Medicae enthalten, Und in Fünf Decurien eingetheilet, Dem Publico zum Besten herausgegeben. Fünffter Theil. Halle im Magdeburgischen: Renger.

Metzger, Johann Daniel (1781): Medicinisch-gerichtliche Beobachtungen. Erster Jahrgang. Königsberg: F. D. Wagner \& R. G. Dengel.

Petermann, Andreas (1709): Casuum medico-legalium Decas II. Herausgegeben von Dessen Sohne/ Benj. Petermannen / Practico daselbst. Leipzig.

Ruef, Johann Caspar (1777): Unterricht von Criminalfällen, und wie sich ein Arzt in Abgebung seines Gutachtens hierüber zu verhalten habe. Nebst einem Anhang von der Hornviehseuche. Nürnberg: Wolfgang Schwarzkopf.

Stahl, Georg Ernst (1733): Collegivm Casvale Magnvm, oder Sechs und siebentzig Practische Casus, welche von Anno 1705. biß 1707. als Professor Ordinarius auf der Universität Halle einem gewissen Numero Studiosorum mit gründlicher Resolution und treuer Eröffnung vieler besondern Practischen Cau-telen Lateinisch in die Feder dictirt, Nunmehro ins Deutsche übersetzt Von D. Johann Storchen, alias Hulderico Pelargo [...]. Leipzig: Caspar Jacob Eissel.

Troppanneger, Christian Gottlieb (1733): Decisiones Medico-Forenses. Sowohl dessen eigene, und zwar die meisten Judicis, also auch anderer, und unterschiedlicher Juristisch und Medicinischer Facultäten Urthel und Responsa, Uber Siebentzig rare und zum Theil schwere Casus, Sonderlich De Lethalitate Vulnerum. [...] Dresden.

Zittmann, Friedrich (1706): Medicina Forensis. Das ist Eröffnete Pforte zur Medicin und Chirurgie. Anweisende Hochlöbl. Medicinischen Facultät zu Leipzig hoch vernünfftig ertheilte Aussprüche und Responsa, Uber allerhand schwere/ zweiffelhaffte und seltene / von Anno 1650. biß 1700 vorgekommene und in die Medicin, auch Chirurgie lauffende Fragen und Fälle / [...] Frankfurt a. M.

Zittmann, Friedrich (1706): Medicina Forensis h(ic).e(st). Responsa Facultatis MedicA Lipsiensis Ad QuAEstiones casus medicinales Ab Anno MDCL. usque MDXX In ufum communem evulgata A D. JOH. FRIDER.ZITTMANNO. Sereniff. Potentiff. Regis Polon. Ge Medica Caftrenfi. Cum ejus Præfatione \& Indic Francofurti ad Mœnum Sumptibus SOCIETATIS ANNO M DCC VI. 


\section{Onlinequellen}

Retrospektive Digitalisierung wissenschaftlicher Rezensionsorgane und Literaturzeitschriften des 18. und 19. Jahrhunderts aus dem deutschen Sprachraum: http://www.ub.unibielefeld.de/diglib/aufklaerung/

\section{Literatur}

Ammon, Ulrich (2015): Die Stellung der deutschen Sprache in der Welt. Berlin u. a.:

De Gruyter.

Behrens, Rudolf \& Carsten Zelle (Hrsg.) (2012): Der ärztliche Fallbericht. Epistemische Grundlagen und textuelle Strukturen dargestellter Beobachtung. Wiesbaden: Harrasowitz.

Berndt, Frauke \& Daniel Fulda (Hrsg.) (2012): Die Sachen der Aufklärung. Hamburg: Meiner.

Daston, Lorraine (2011a): Observation. In: Susan Dackerman (Hrsg.), Prints and the Pursuit of Knowledge in Early Modern Europe. Cambridge, New Haven: Harvard University Art Museums, 126-133.

Daston, Lorraine (2011b): The Empire of Observation 1600-1800. In: Lorraine Daston \& Elizabeth Lunbeck (Hrsg.), Histories of Scientific Observation. Chicago: The University of Chicago Press, 81-113.

Daston, Lorraine \& Elizabeth Lunbeck (Hrsg.) (2011): Histories of Scientific Observation. Chicago: The University of Chicago Press.

Düwell, Susanne \& Nicolas Pethes (Hrsg.) (2014): Fall. Fallgeschichte. Fallstudie. Theorie und Geschichte einer Wissensform. Frankfurt am Main: Campus.

Fischer-Homberger, Esther (1988): Medizin vor Gericht. Zur Sozialgeschichte der Gerichtsmedizin. Darmstadt: Luchterhand.

Geyer-Kordesch, Johanna (1990): Medizinische Fallbeschreibungen und ihre Bedeutung für die Wissensreform des 17. und 18. Jahrhunderts. In: Medizin, Gesellschaft und Geschichte 9, 7-19.

Götz, Ursula (2011): Zur Syntax von Titelblättern des 16. Jahrhunderts. In: Franz Simmler \& Claudia Wich-Reif (Hrsg.), Geschichte der Gesamtsatzstrukturen vom Althochdeutschen bis zum Frühneuhochdeutschen. Bern u. a.: Peter Lang, 67-95.

Heinemann, Wolfgang (2000): Textsorte - Textmuster - Texttyp. In: Armin Burkhardt, Hugo Steger \& Herbert Ernst Wiegand (Hrsg.), Text- und Gesprächslinguistik. Ein internationales Handbuch zeitgenössischer Forschung (HSK 16.1). Berlin, New York: De Gruyter, 507-523.

Klein, Wolf Peter (2011a): Deutsch statt Latein! Zur Entwicklung der Wissenschaftssprachen in der frühen Neuzeit. In: Wieland Eins, Helmut Glück \& Sabine Pretschler (Hrsg.), Wissen schaffen - Wissen kommunizieren. Wissenschaftssprachen in Geschichte und Gegenwart. Wiesbaden: Harrasowitz, 35-47.

Klein, Wolf Peter (2011b): Die deutsche Sprache in der Gelehrsamkeit der frühen Neuzeit. Von der lingua barbarica zur HaubtSprache. In: Herbert Jaumann (Hrsg.), Diskurse der Gelehrtenkultur in der Frühen Neuzeit. Berlin, New York: De Gruyter, 465-516.

Kretzenbacher, Heinz L. \& Harald Weinrich (Hrsg.) (1995): Linguistik der Wissenschaftssprache. Berlin, New York: De Gruyter. 
Kretzenbacher, Heinz L. (1995): Wie durchsichtig ist die Wissenschaftssprache? In: Heinz L. Kretzenbacher \& Harald Weinrich: Linguistik der Wissenschaftssprache. Berlin, New York: De Gruyter, 15-39.

Lindner, Bettina (2015): Quotations from $17^{\text {th }}$ and $18^{\text {th }}$ Century Medical Case Reports. In: Jenny Arendholz, Wolfram Bublitz \& Monika Kirner-Ludwig (Hrsg.), The pragmatics of quoting now and then (Topics in English Linguistics 89). Berlin, New York: De Gruyter, 401-418.

Lindner, Bettina (2018): Medizinische Gutachten des 17. und 18. Jahrhunderts.

Sprachhistorische Untersuchungen zu einer Textsortenklasse (Lingua Academica 2). Berlin, New York: De Gruyter.

Lorenz, Maren (1999): Die Normierung des Individuums in Gerichtsmedizin und Psychiatrie der Aufklärung. Hamburg: Hamburger Edition.

Müller, Irmgard \& Heiner Fangerau (2010): Protokolle des Unsichtbaren: Visa reperta in der gerichsmedizinischen Praxis des 18. und 19. Jahrhunderts und ihre Rolle als Promotoren pathologisch-anatomischen Wissens. In: Medizinhistorisches Journal 45, 265-292.

Polenz, Peter von (2013): Deutsche Sprachgeschichte vom Spätmittelalter bis zur Gegenwart. Bd. II: 17. und 18. Jahrhundert. 2. Aufl. Berlin, Boston: De Gruyter.

Pomata, Gianna (2010): Sharing Cases: The Observationes in Early Modern Medicine. In: Early Science and Medicine 15, 193-236.

Pomata, Gianna (2011): Observation Rising: Birth of an Epistemic Genre 1500-1600. In: Lorraine Daston \& Elizabeth Lunbeck (Hrsg.), Histories of Scientific Observation. Chicago: The University of Chicago Press, 45-80.

Pörksen, Uwe (1983): Der Übergang vom Gelehrtenlatein zur deutschen Wissenschaftssprache. Zur frühen deutschen Fachliteratur und Fachsprache in den naturwissenschaftlichen und mathematischen Fächern (ca. 1500-1800). In: Zeitschrift für Literaturwissenschaft und Linguistik 52/53, 227-258.

Pörksen, Uwe (2004): Deutsche Sprachgeschichte und die Entwicklung der Naturwissenschaften. Aspekte einer Geschichte der Naturwissenschaftssprache und ihrer Wechselbeziehungen zur Gemeinsprache. In: Werner Besch et al. (Hrsg.), Sprachgeschichte. Ein Handbuch zur Geschichte der deutschen Sprache und ihrer Erforschung (HSK 2.4), 2. Aufl. Berlin, New York: De Gruyter, 85-101.

Ruisinger, Marion Maria (2008): Patientenwege. Die Konsiliarkorrespondenz Lorenz Heisters (1683-1758) in der Trew-Sammlung Erlangen. Stuttgart: Franz Steiner.

Schiewe, Jürgen (1996): Sprachenwechsel - Funktionswandel - Austausch der Denkstile. Die Universität Freiburg zwischen Latein und Deutsch. Tübingen: Niemeyer.

Schnalke, Thomas (1995): Natur im Bild. Anatomie und Botanik in der Sammlung des Nürnberger Arztes Christoph Jacob Trew. Eine Ausstellung aus Anlaß seines 300. Geburtstages, 8. November-10. Dezember 1995. Erlangen: Universitätsbibliothek.

Schnalke, Thomas (1997): Medizin im Brief. Der städtische Arzt des 18. Jahrhunderts im Spiegel seiner Korrespondenz. Stuttgart: Franz Steiner.

Schulz, Matthias (2012): Fraktur und Antiqua in deutschsprachigen gedruckten Texten des 17. Jahrhunderts. In: Sprachwissenschaft 37 (4), 423-456.

Stolberg, Michael (2003): Formen und Strategien der Autorisierung in der frühneuzeitlichen Medizin. In: Wulf Oesterreicher, Gerhard Regn \& Winfried Schulze (Hrsg.), Autorität der Form - Autorisierung - Institutionelle Autorität. Münster: LIT, 205-218.

Stolberg, Michael (2007): Formen und Funktionen medizinischer Fallberichte in der Frühen Neuzeit (1500-1800). In: Johannes Süßmann, Susanne Scholz \& Gisela Engel (Hrsg.), Fallstudien: Theorie - Geschichte - Methode. Berlin: Trafo, 81-95

Wübben, Yvonne \& Carsten Zelle (Hrsg.) (2013): Krankheit schreiben. Aufzeichnungsverfahren in Medizin und Literatur. Göttingen: Wallstein. 